\title{
Identificación del modelo pedagógico - matemático en la práctica docente
}

\section{identification of the pedagogical - mathematical model in teaching practice}

Narcisa de Jesús Sánchez Salcán. ${ }^{1}$, Fabian Patricio Londo Yachambáy ${ }^{2}$,

\begin{abstract}
.
We are all concerned about the low performance to students in the learning of mathematics. The student assessments at national and international level exposed profound deficiencies, taking as an antecedent that Ecuador failed in Mathematics in the international assessment of PISA (Program for International Student Assessment), for that reason, the present research aims to identify the pedagogical model that are employing teachers in the area of mathematics to impart their classes to students of Basic Education level higher. The type of research is descriptive, with a non-experimental research design. The population under study are teachers who work in the educational units of the Chambo district Riobamba. The techniques used to collect the data were: interview, observation and survey. The results obtained, it was concluded that, in the educational institutions designed for the study, some teachers privilege the explanation of algorithms based on learning stages or steps in the resolution of problems, without giving rise to what is most important: your comprehension, only should memorize steps, equations, or procedures, which corresponds to a traditional pedagogical model and behavioral therapy.
\end{abstract}

Keywords: Enseñanza, aprendizaje, matemática, docencia, pedagogía

\section{Resumen.}

Todos estamos preocupados por el bajo desempeño que los estudiantes tienen en el aprendizaje de las matemáticas. Las evaluaciones de los estudiantes a nivel nacional e

1 Universidad Nacional de Chimborazo. Facultad de Ciencias de la Educación Humanas y

Tecnologías nsanchez@unach.edu.ec

${ }^{2}$ Escuela Superior Politécnica de Chimborazo. Facultad de Administración de Empresas. flondo@espoch.edu.ec 
internacional exponen profundas deficiencias, teniendo como antecedente que Ecuador reprobó en Matemáticas en la evaluación internacional PISA (Programme For International Student Assessment), por tal motivo la presente investigación pretende identificar el modelo pedagógico que están empleando los docentes del área de Matemática al impartir sus clases a los estudiantes del nivel de Educación Básica Superior. El tipo de investigación es descriptivo, con un diseño de investigación no experimental. La población objeto de estudio son los docentes que laboran en las Unidades Educativas del distrito Chambo Riobamba. Las técnicas empleadas para la recolección de los datos fueron: entrevista, observación y encuesta. De los resultados obtenidos se concluye que, en las instituciones educativas destinadas para el estudio, algunos docentes privilegian la explicación de algoritmos basados en aprender etapas o pasos en la resolución de problemas, sin dar lugar a lo más importante: su comprensión, solo deben memorizar pasos, ecuaciones o procedimientos, lo que corresponde a un modelo pedagógico tradicional y conductista.

Palabras claves: teaching, learning, mathematics, teaching, pedagogy

\section{Introducción.}

Todos estamos preocupados por el bajo desempeño que los estudiantes tienen en el aprendizaje de las matemáticas. Las evaluaciones de los estudiantes a nivel nacional e internacional exponen profundas deficiencias, teniendo como antecedente que Ecuador reprobó en Matemáticas en la evaluación internacional PISA (Programme For International Student Assessment), realizada a los estudiantes de las instituciones educativas fiscales.

Graves dificultades de los estudiantes ecuatorianos para desenvolverse en situaciones que requieren la capacidad de resolver problemas matemáticos arrojaron los resultados de las pruebas PISA-D 2018, en las que el Ecuador participó por primera vez. El 70,9\% de los estudiantes de Ecuador no alcanzó en Matemáticas el nivel 2, categorizado como el nivel de desempeño básico. El desempeño promedio de Ecuador fue de 377 sobre 1.000. (Publicado en el diario el Universo con fecha 26 de febrero del 2019).

Estos resultados plantean un llamado de atención acerca de cómo fortalecer lo que está pasando en todas las aulas del país, y especialmente en las escuelas de contextos de mayor pobreza. Las investigaciones revelan que en las clases de matemática los estudiantes dedican el tiempo a hacer cuentas y resolver ejercicios de manera mecánica, sin comprender el sentido ni la relevancia de lo que están trabajando.

El mito de que las Matemáticas son difíciles es falso, lo primero es trabajar en la actitud por el gusto de la matemática con los estudiantes, y como segundo paso es cambiar la forma de enseñar, las matemáticas hay que explicarles de lo real a lo abstracto y para ello se debe plantear juegos y hacer magia.

La presente investigación relaciona los resultados obtenidos de varias investigaciones que sirvieron de base y están relacionados con el objeto de estudio. 
A nivel internacional la investigación: Análisis de los modelos pedagógicos implementados en tres instituciones educativas del sector oficial de la ciudad de Santiago de Cali realizada por Noel Estupiñan, el objetivo principal fue determinar las características generales del modelo de escuela (tradicional o nueva) prevaleciente en las instituciones educativas Santa Cecilia, INEM Jorge Isaac y Libardo Madrid Valderrama, del sector oficial de la ciudad de Santiago de Cali. Luego del análisis y procesamiento de la información concluye que: El proceso enseñanza-aprendizaje es dirigido por el docente, quien considera que los estudiantes manejan preconceptos de cada uno de los temas a estudiarse durante el año lectivo, la tendencia a desarrollar las clases de manera uniforme, siguiendo siempre los mismos parámetros: utilizar tablero, explicar y dictar. (Estupiñan, 2012).

A nivel nacional la investigación: "Realidad de la práctica pedagógica y curricular en la educación ecuatoriana en los centros educativos de básica y bachillerato de la Unidad Educativa Fe y Alegría “La Dolorosa “ciudad Manta durante el año 2011-2012”, realizada por Galarza Mena, Bethy Arlene, el propósito de la investigación fue dar a conocer las diferentes prácticas pedagógicas que se dan en la educación básica y en el bachillerato en los centros educativos de nuestro país, en particular en la Unidad Educativa "La Dolorosa de la ciudad de Manta". Luego de seguir la secuencia del proceso, aplicando todos los procedimientos recomendados y utilizando la encuesta y la observación como técnicas e instrumentos de recolección de datos, a docentes y estudiantes, se obtuvo resultados válidos y confiables que luego de analizados e interpretados permitieron llegar a conclusiones en el estudio como: el modelo educativo que aplican los docentes en su clase no es definido, es decir la institución carece de una modelo específico oficial, que dirija el proceso de enseñanza aprendizaje. Este resultado es de mucha importancia, porque en todo proceso se exige una dirección, más el proceso pedagógico, que por sus múltiples funciones y condicionamientos es complejo, necesita ser pensado, diseñado con anterioridad, de manera que se pueda predecir las modificaciones y transformaciones propiciando su desarrollo. (Galarza \& Arlene. (2012).

En algunas instituciones educativas como en el caso de Colombia, el problema es que siguen utilizando un modelo tradicional, siendo el docente quien dirige el proceso de enseñanza aprendizaje, y es el que considera que sus estudiantes manejan preconceptos de cada uno de los temas a estudiarse en el año lectivo. Las clases son tradicionales, en la pizarra y sin la utilización de materiales innovadores para que el aprendizaje sea significativo. En el proceso de enseñanza no se desarrollan estrategias pedagógicas y didácticas que permitan la generación del conocimiento por parte de los estudiantes. Si bien se ha procurado incorporar las tecnologías de información y comunicación, estas son subutilizadas y no existe una política institucional clara para que sean implementadas de manera continua en los procesos de enseñanza y aprendizaje (Estupiñán, 2012).

Los datos anteriores confirman lo señalado por Flores (2005), en el sentido que los docentes son híbridos en cuestiones de pedagogía; en el desarrollo de su hacer profesional, toman de una $\mathrm{u}$ otra corriente lo que les sirva para el momento, sin asumir las consecuencias epistemológicas, éticas, psicológicas o políticas que están en el fondo de ellas.

Así mismo, en Ecuador ciertas Instituciones Educativas presentan dificultades en la enseñanza y aprendizaje de las ciencias, registrando resultados académicos bajos lo que se derivan en un supuesto a la falta de identificación con el modelo pedagógico institucional. 
Por ello surge la siguiente interrogante: ¿Qué Modelo Pedagógico aplican los docentes que imparten clases a los estudiantes de Educación básica Superior dentro de su práctica docente en el área de Matemática de las Unidades Educativas de Chambo-Riobamba?.

El modelo pedagógico aplicable en las instituciones educativas a nivel nacional es el constructivismo, modelo que centra su atención en el estudiante siendo el principal protagonista en el proceso de enseñanza-aprendizaje, y el elemento más importante e imprescindible en la educación, también hace que el estudiante sea capaz de realizar cosas por sí solo, tenga un aprendizaje significativo, cree un pensamiento más crítico, sea más participativo en las clases y no tenga miedo a expresarse.

Sin embargo, este modelo no es practicado por todos los docentes, algunos solo se limitan a impartir sus clases, no utilizan modelos y técnicas de enseñanza y aprendizaje, repercutiendo negativamente en la labor que se desarrolla en clases, perjudicando el rendimiento académico de los estudiantes.

Los modelos pedagógicos son una forma de concebir la práctica de los procesos formativos. Comprende los procesos relativos a las cuestiones pedagógicas de cómo se aprende, cómo se enseña, las metodologías más adecuadas para la asimilación significativa de los conocimientos, habilidades y valores, las consideraciones epistemológicas en torno a la pedagogía, las aplicaciones didácticas, el currículo y la evaluación de los aprendizajes, es indispensable conocer las características que subyacen a los diferentes modelos de la educación ya que esto, además de permitir comprender lo que ha pasado en la educación y hacia dónde va, permitirá realizar un análisis de la práctica docente, del planteamiento metodológico, de las estrategias de aprendizaje utilizadas, etc. Hechos que siempre están presentes en la planeación didáctica, he ahí la importancia de investigar los modelos pedagógicos.

Por todo lo antes mencionado es importante realizar éste estudio, por conocer lo que está ocurriendo dentro de las aulas de clase y saber exactamente cuál es el modelo pedagógico que utilizan los docentes al impartir las clases en las instituciones educativas en estudio y saber sus repercusiones en el sistema educativo, sabiendo que los modelos pedagógicos es una herramienta necesaria en la labor docente.

Ésta investigación servirá de orientación para docentes y estudiantes, brindando motivación para que otros investigadores continúen profundizando el estudio con nuevas exploraciones. Además, con los resultados obtenidos se pueden motivar a discutir, cuestionar, confrontar y reflexionar sobre los componentes del modelo pedagógico: contenidos, metodologías, recursos, evaluación, docente vs estudiante involucrados en el quehacer educativo, como también se podrá constatar la realidad de la práctica educativa al interior de las aulas de clase enfrentada a los ideales establecidos desde los escritorios y plasmados en documentos.

Igualmente, los resultados obtenidos en el desarrollo de la presente investigación aspiran a reforzar los estudios que con antelación se han realizado al respecto y de esta manera contribuir al mejoramiento de las políticas, planes y proyectos educativos que se elaboran a nivel nacional, regional y local.

El objetivo principal de esta investigación fue determinar el Modelo Pedagógico empleados por los docentes que imparten clases a los estudiantes del nivel de Educación, Básica 
Superior, dentro de su práctica docente en el área de Matemática de las Unidades Educativas del distrito Chambo-Riobamba.

\section{Metodología}

El presente artículo es una investigación realizada a partir de un enfoque cuantitativo, posee un carácter descriptivo porque se observó, registró y describió el modelo pedagógico que emplean los docentes en la enseñanza aprendizaje de la matemática.

Se desarrolló dentro del paradigma de la investigación - acción, pues ella permite analizar situaciones vividas por parte de los miembros de la comunidad educativa y avanzar en la construcción de soluciones a las necesidades presentadas en la práctica pedagógica.

Kemmis \& Taggart (como se citó en Benjumeda, 2012), el proceso puede resumirse en las siguientes fases:

- Diagnóstico y reconocimiento de las situación actual

- Desarrollo de un plan de acción, críticamente informado, para mejorar aquello que ya está ocurriendo

- Actuación para poner el plan en práctica y la observación de sus efectos en el contexto que tiene lugar la reflexión en torno a los efectos como base para una nueva planificación.

El diseño de la investigación es no experimental, porque el estudio se realizó sin manipular deliberadamente las variables.

Como nos indica Mateo (2000), las metodologías ex-post-facto o no experimental son las más utilizadas en el ámbito educativo, proporcionándonos técnicas para describir la realidad, analizar relaciones, categorizar, simplificar y organizar las variables que configuran el objeto de estudio.

El diagrama simbólico es: (Sánchez-Reyes: 62) M O Dónde: M es la muestra y O la observación relevante sobre los modelos pedagógicos

Para iniciar se realizó una investigación de tipo bibliográfico sobre los modelos pedagógicos y teorías curriculares. Posteriormente se realizó una investigación de campo en las instituciones educativas del distrito Chambo -Riobamba específicamente en las unidades educativas: Yaruquies, Pedro Vicente Maldonado, Nicanor Larrea y Colegio Bachillerato Chambo, con la intención de establecer el modelo pedagógico empleado por los docentes del área de matemática

En la parte cuantitativa del estudio se analizó todos los resultados obtenidos de la ficha de entrevista, ficha de observación y la encuesta, que fueron aplicados a los docentes de las instituciones educativas antes mencionadas, para conocer los modelos pedagógicos que emplean los docentes en la práctica pedagógica.

Los métodos utilizados fueron el método descriptivo, deductivo e inductivo.

Se seleccionó como universo de estudio a los docentes que dictan clases de matemática en el nivel de Educación, Básica Superior de las instituciones educativas de los distritos ChamboRiobamba, siendo un total de 146 docentes. 
El muestreo fue no probabilístico de tipo intencional porque se escogió a los individuos de la muestra según las circunstancias de mayor facilidad, siendo considerados 28 docentes del área de matemática.

Tabla 1. Muestra

\begin{tabular}{llc}
\hline Distrito & Unidad educativa & Número de docentes \\
\hline Chambo-Riobamba & Pedro Vicente Maldonado & 15 \\
& Yaruquies & 3 \\
& Dr. Nicanor Larrea & 5 \\
& Colegio de Bachillerato Chambo & 5 \\
& & TOTAL \\
\hline
\end{tabular}

Fuente: Datos entregados por la Dirección distrital Chambo-Riobamba

En base al propósito del estudio como instrumentos utilizados para la recolección de los datos fueron: la ficha de observación, la ficha de entrevista y el cuestionario dirigida a los docentes del distrito Chambo-Riobamba que laboran en las instituciones educativas: Pedro Vicente Maldonado, Yaruquies, Dr. Nicanor Larrea y Colegio de Bachillerato Chambo, con la finalidad de conocer cuál o cuáles son los modelos pedagógicos que utilizan los docentes en la práctica pedagógica.

Cabe mencionar que los instrumentos fueron validados por algunos docentes de la carrera de licenciatura en Pedagogía de las Ciencias Experimentales: Matemáticas y la Física, para su posterior aplicación.

\section{Resultados y Discusión}

Los modelos pedagógicos cobran un papel trascendental, ya que el uso de un buen modelo se convierte en una herramienta de apoyo para el docente, permitiendo regular y mejorar el proceso de enseñanza-aprendizaje, siendo las autoridades a nivel educativo las que deben preocuparse para mejorar la calidad de educación en nuestro país.

Considerando a los modelos educativos como un factor fundamental para el análisis y la reflexión de las metodologías y recursos didácticos utilizados, se aplicó una entrevista estructurada a los rectores de las instituciones educativas del distrito Chambo-Riobamba siendo: Pedro Vicente Maldonado, Yaruquies, Dr. Nicanor Larrea y colegio de Bachillerato Chambo, así también se aplicó la ficha de observación y el cuestionario a los docentes de matemática de dictan clases en el nivel de Educación Básica Superior de las instituciones educativas antes mencionadas.

De manera resumida las respuestas de la ficha de entrevista estructurada aplicada a los rectores de las instituciones educativas fueron:

- ¿Cuál es el modelo pedagógico que aplican en su institución? 
Toda institución educativa trata de cumplir con las disposiciones emanadas por el organismo rector que es el Ministerio de Educación, de acuerdo al proyecto Educativo Institucional y al proyecto curricular Institucional se trabaja con dos modelos: el constructivismo y el socio crítico propendiendo el aprendizaje significativo.

- ¿Está de acuerdo con el modelo pedagógico empleado?

$\mathrm{Si}$, todos los modelos pedagógicos tienen un procedimiento y recomendaciones que les entregan a los docentes, para alcanzar aprendizajes significativos en los estudiantes, todo modelo pedagógico se lo debe adaptar de acuerdo a las necesidades que tiene el estudiantado y la sociedad.

- ¿El modelo pedagógico institucional está relacionado con las políticas, principios, misión y visión de la institución?

El modelo pedagógico institucional ésta relacionado con las políticas, principios, misión y visión de la institución, focalizado en desarrollar las habilidades cognitivas y actitudinales en los estudiantes al máximo.

- ¿El modelo pedagógico orientador de su institución responde a las necesidades e intereses de la comunidad educativa?

Lamentablemente no, porque dentro de éste modelo pedagógico, está el desarrollo de las destrezas con criterio de desempeño, que tiene que alcanzar el estudiante dependiendo de la asignatura, por ejemplo en las matemáticas, física, química entre otras, pero como se fomenta esas destrezas si no hay el equipamiento técnico necesario como el de disponer de laboratorios adecuados, lo cual hace imposible conseguir un aprendizaje duradero y sobre todo significativo.

- ¿Considera que se debe realizar mejoras al modelo pedagógico? y ¿cuáles serían?

Por supuesto que sí, todos estamos sujetos a cambios, estamos buscando actualizaciones incluso tratamos de ver que estos modelos pedagógicos se acoplen a nuestro medio, no que simplemente se impongan, los docentes somos los que más exigimos para que exista un cambio en el sistema educativo.

Además, los modelos pedagógicos no están escritos en piedra, son principios que el docente lo adapta, son recomendaciones que hay que adaptarlas a las necesidades del estudiante. Con el modelo constructivista- social el docente trabaja con una gama de metodologías y técnicas para facilitar el aprendizaje, de acuerdo a las necesidades que va teniendo cada estudiante, pero cada uno tiene diferentes procesos de aprendizaje y más bien es el aprendizaje que se tiene que adaptar a las necesidades del estudiante.

- ¿La institución realiza seguimiento a los docentes, para verificar el desarrollo del modelo pedagógico institucional?

El vicerrectorado es el organismo encargado de supervisar todo el proceso del aprendizaje, pero también se tiene la junta académica, siendo un organismo que integran todos los coordinadores pedagógicos de todas las áreas. La metodología empleada para el monitoreo en el proceso de aprendizaje es la visita a las horas de clase del docente conjuntamente con el Sr rector, vicerrector y la junta académica, y en base a lo observado y a los resultados obtenidos en el rendimiento académico de los estudiantes se hace la recomendación para el fortalecimiento en el aprendizaje del estudiante. 
Además, se busca que cada día los docentes estén actualizados en sus conocimientos, a base de los cursos de capacitación ya sea del Ministerio de Educación o de otros entes públicos. De la entrevista realizada a los rectores de las unidades educativas en estudio, ellos mencionan que el modelo pedagógico aplicado en las instituciones educativas es el constructivismo siendo un buen modelo pedagógico, pero existen diversas falencias por no contar con el equipamiento tecnológico necesario, el contexto socioeconómico de los padres no es bueno, pese a ello tratan de acoplar con lo que cuentan para alcanzar la meta propuesta. Por lo antes mencionado es necesario tomar en consideración que para tener un buen modelo educativo y pedagógico se debe tomar en cuenta las siguientes componentes:

- Aprender haciendo. El aprendizaje debe ser basado en problemas, casos y/o proyectos, se debe fomentar actitudes estratégicas, más que enseñar estrategias concretas o discursos sobre estrategia.

- Esencializar el currículum. Se debe tomar en consideración que la selección del currículum escolar contemporáneo no debe ser extenso, se requiere que sea de calidad y no de cantidad.

- Currículum basado en casos, problemas y proyectos. El trabajo por problemas, casos o proyectos requiere elaborar un currículum en el que la mayoría de los problemas se refieran a situaciones novedosas, es decir, situaciones en las que no parece obvia la solución ni las peculiaridades del problema. El currículo tiene que poner al estudiante en situaciones de desafío; la primera tarea consiste en buscar el conocimiento adecuado y relevante para identificarlas, entenderlas y afrontarlas.

- Promover la didáctica invertida (Flipped Classroom), las redes sociales virtuales y las plataformas digitales. Aprovechar al máximo los recursos digitales y las redes sociales, plataformas, laboratorios, enciclopedias y talleres virtuales, herramientas de diseño digital, espacios virtuales de cooperación, plataformas e instrumentos de realidad aumentada, herramientas de fabricación en 3D con el propósito de fomentar la competencia digital como usuarios activos y creativos de todos los aprendices.

- Primar la cooperación y fomentar el clima de confianza. La cooperación aparece como la estrategia pedagógica privilegiada tanto para el desarrollo de los componentes cognitivos como de los componentes emotivos y actitudinales de las competencias (Darling-Hammond, 2010).

- Fomentar la metacognición. Promover y estimular la metacognición como medio para desarrollar la capacidad de autonomía y autorregulación del aprendizaje y del desarrollo.

- Apostar decididamente por la evaluación formativa. Esto implica fomentar la evaluación que ayuda a mejorar los procesos de aprendizaje, es decir, estimular los procesos de auto y coevaluación entre pares, la utilización de procedimientos como el portafolio, la tutorización cercana o el reconocimiento del error como ocasión de aprendizaje.

- Estimular la función tutorial del docente. Ante estos nuevos retos, los docentes en la era digital son más necesarios que nunca, no precisamente para transmitir, sino para ayudar a aprender, a construir el propio conocimiento y a autorregular el propio aprendizaje de cada estudiante.

Por otra parte, se aplicó una encuesta a los 28 docentes de matemáticas de las instituciones educativas en estudio, la cual constó de 40 preguntas evidenciándose en cada pregunta el tipo 
de modelo pedagógico. Con los datos obtenidos se realizó una interpretación clara del modelo pedagógico empleado por los docentes obteniéndose los siguientes resultados:

A las respuestas señaladas en el formato de la encuesta, se asignó los siguientes valores:

- Nada: 0

- Un poco: 1

- Bastante: 2

- Totalmente: 3

En cada tabla, de acuerdo con el número de la pregunta, se asignó el valor que corresponde a la respuesta dada:

Tabla 2. Tabla de puntajes del Modelo Pedagógico Tradicional.

\begin{tabular}{llllllllllll}
\hline $\begin{array}{l}\mathrm{N}^{\circ} \\
\text { Preguntas }\end{array}$ & 1 & 5 & 9 & 13 & 17 & 21 & 25 & 29 & 33 & 37 & Total \\
\hline $\begin{array}{l}\text { Valores } \\
\text { asignados }\end{array}$ & 2 & 3 & 1 & 2 & 3 & 0 & 2 & 1 & 1 & 1 & 15 \\
\hline
\end{tabular}

Fuente: Datos obtenidos de la encuesta aplicada a los docentes de Matemática

Tabla 3. Tabla de puntajes del Modelo Pedagógico Conductista.

\begin{tabular}{llllllllllll}
\hline $\begin{array}{l}\mathrm{N}^{\mathrm{o}} \\
\text { Preguntas }\end{array}$ & 2 & 6 & 10 & 14 & 18 & 22 & 26 & 30 & 34 & 38 & Total \\
\hline $\begin{array}{l}\text { Valores } \\
\text { asignados }\end{array}$ & 2 & 3 & 1 & 3 & 3 & 2 & 3 & 2 & 3 & 2 & 24 \\
\hline
\end{tabular}

Fuente: Datos obtenidos de la encuesta aplicada a los docentes de Matemática

Tabla 4. Tabla de puntajes del modelo Pedagógico Constructivista

\begin{tabular}{cccccccccccc}
\hline $\begin{array}{c}\mathrm{N}^{\mathrm{o}} \\
\text { Preguntas }\end{array}$ & 3 & 7 & 11 & 15 & 19 & 23 & 27 & 31 & 35 & 39 & Total \\
\hline $\begin{array}{c}\text { Valores } \\
\text { asignados }\end{array}$ & 1 & 0 & 2 & 1 & 1 & 0 & 1 & 1 & 2 & 1 & 10 \\
\hline
\end{tabular}

Fuente: Datos obtenidos de la encuesta aplicada a los docentes de Matemática

Tabla 5. Tabla de puntajes del modelo Pedagógico Social

\begin{tabular}{cccccccccccc}
\hline $\begin{array}{c}\mathrm{N}^{\mathbf{0}} \\
\text { Preguntas }\end{array}$ & 4 & 8 & 12 & 16 & 20 & 24 & 28 & 32 & 36 & 40 & Total \\
\hline $\begin{array}{c}\text { Valores } \\
\text { asignados }\end{array}$ & 1 & 3 & 2 & 0 & 1 & 2 & 2 & 1 & 3 & 2 & 17 \\
\hline
\end{tabular}

Fuente: Datos obtenidos de la encuesta aplicada a los docentes de Matemática

Cada una de las anteriores tablas corresponde a las características de las prácticas pedagógicas de un determinado modelo pedagógico así:

Tabla $\mathrm{N}^{\circ}$ 2: Modelo Pedagógico Tradicional.

Tabla $\mathrm{N}^{\mathrm{o}}$ 3: Modelo Pedagógico Conductista

Tabla $N^{\circ}$ 4: Modelo Pedagógico Constructivista

Tabla N ${ }^{\circ}$ 5: Modelo Pedagógico Social. 
Con base en los puntajes de cada tabla, sus resultados se interpretan como nada, un poco, bastante o totalmente perteneciente a un Modelo Pedagógico determinado, así:

0: Nada (Tradicional, Conductista, Desarrollista o Social)

De 1 a 10: Un poco (Tradicional, Conductista, Desarrollista o Social)

De 11 a 20: Bastante (Tradicional, Conductista, Desarrollista o Social)

De 21 a 31: Totalmente: (Tradicional, Conductista, Desarrollista o Social)

Tabla 6. Resultados del Modelo Pedagógico

\begin{tabular}{cll}
\hline Puntaje & \multicolumn{1}{c}{ Escala } & \multicolumn{1}{c}{ Modelo Pedagógico } \\
\hline 15 & Bastante & Tradicional \\
24 & Totalmente & Conductista \\
10 & Un poco & Constructivista \\
17 & Bastante & Social \\
\hline
\end{tabular}

Fuente: Resumen de los datos obtenidos de las tablas: 2,3,4,5

Los datos demuestran que en la mayoría de las instituciones educativas el docente no sustenta su práctica pedagógica en el modelo pedagógico descrito por el Ministerio de Educación como es el constructivismo. Según los resultados el Modelo Pedagógico predominante es el modelo conductista, por lo que es necesario la puesta en marcha de programas masivos de capacitación dirigidos a la gran mayoría de los docentes, en relación al modelo pedagógico, al empleo de nuevas metodologías que vayan de acuerdo con las necesidades de la institución y con el desarrollo tecnológico de la actualidad.

Los docentes encuestados de las cuatro instituciones educativas, tienen claridad sobre el papel de importancia que incumbe al estudiante en el proceso educativo. Además, asumen una posición de entender las características de los estudiantes, coincidente con los postulados de Piaget, Montessori, Ausubel, Merani, Wallon, Zuleta y De Zubiria, pero esto es contradictorio con los resultados obtenidos dentro de la labor docente.

Algo fundamental que se debe tomar en consideración en el proceso de enseñanza aprendizaje es la motivación en los estudiantes hacia el aprendizaje de la matemática, puesto que mientras ellos se divierten también aprenden, y para tener interés por algo se debe incentivar sobre todo hacia la experimentación.

De las observaciones de clase realizadas a los docentes de matemática se evidenció que, en las cuatro unidades educativas, la mayoría de los docentes tienen dominio de la asignatura, son abiertos a cuestionamientos y discusiones dirigidas, pero en las clases no emplean material didáctico pertinente, siendo indispensable que el docente utilice material didáctico adecuado acorde a las necesidades del estudiante; también utilice actividades dinámicas que motive a los estudiantes a aprender y a la vez refuerce lo aprendido.

En algunos casos se observó que el docente al momento de impartir sus clases utiliza el libro para realizar el dictado de la temática tratada; en otros casos el docente utiliza la pizarra al momento de impartir sus conocimientos, muy pocos docentes emplean los laboratorios, y las 
tecnologías de la comunicación e información, sabiendo que es importante combinar el saber teórico con la utilidad práctica de los saberes.

El tipo de evaluación que aplican los docentes a los estudiantes en el aula, es abordado desde el modelo conductista, es decir la evaluación se centra en los resultados, el cual es medido a través de exámenes, para determinar si se cumplió o no con los objetivos propuestos.

Una de las preguntas que se realizó tanto a directivos como a los docentes de las instituciones educativas en estudio fue ¿Qué modelo pedagógico orienta la educación ecuatoriana? la respuesta fue de asombro e incertidumbre para algunos, mientras que otros supieron manifestar que el modelo pedagógico es el constructivista.

Según la reforma educativa el modelo que debe predominar en las instituciones educativas a nivel nacional es el modelo constructivista, por lo que los docentes deben reflexionar sobre el uso de la metodología del aprender - haciendo y los procesos de enseñanza de las habilidades cognitivas de más alto orden, formar personas por una parte, con mentalidad abierta, poseedores de un pensamiento crítico-reflexivo-sistemático que sean capaces de movilizar sus estructuras de pensamiento y adaptarse a los constantes cambios que se dan en el medio, y por otra, capaces de interpretar el mundo desde sus propias percepciones y mediante la vivencia y la experiencia que se deriva de un contacto directo con la realidad.

El enfoque constructivista está centrado en la persona, en las experiencias que posee y de las que realiza nuevas construcciones cuando interactúa con el objeto del conocimiento (Piaget) en interacción con otros (Vygotsky) y le resulta significativo (Ausubel) en la medida que estimula lo conceptual (saber), procedimental (saber hacer) y actitudinal (saber ser) (Secretaría de Educación Municipal de Pasto, 2009).

En el proceso enseñanza-aprendizaje, el docente deja de ser transmisor de información, para convertirse en mediador, coordinador, facilitador del aprendizaje del estudiante que asume el rol principal en la generación de sus propios conocimientos. El constructivismo supone también un clima afectivo, armónico, de mutua confianza, que ayude a los estudiantes a vincularse positivamente con el conocimiento, pero de manera particular, en su proceso de adquisición.

Teniendo en cuenta el método empleado por los docentes en el salón de clase, de las cuatro instituciones educativas del distrito Chambo-Riobamba, se evidenció que para la transmisión de saberes específicos predomina el modelo conductista, donde la enseñanza se basa en el cumplimiento de objetivos. Las estrategias de enseñanza deben ser diseñadas de tal manera que estimulen a los estudiantes a observar, analizar, opinar, formular hipótesis, buscar soluciones y descubrir el conocimiento por sí mismos.

Debemos tener claro que la tarea del docente es lograr que los estudiantes sean autónomos, que sean responsables de su propio aprendizaje, que no se limiten sólo a escuchar lo que el docente dice y después lo repita, además de no generar más que el aprendizaje memorístico, y generar apatía hacia el gusto y aprendizaje a las matemáticas, por eso la importancia de utilizar estrategias diversas, siempre encaminadas a lograr un aprendizaje significativo. 
Por otra parte, haciendo referencia a la evaluación de los estudiantes en el logro de aprendizaje de la Matemática, el mayor porcentaje recae en el modelo conductista es decir la evaluación es sumativa a través de exámenes, o preguntas en forma oral, lo que refleja que el docente carece de otras metodologías al momento de evaluar, sabiendo que en nuestro país según las estadísticas dadas por el Ministerio de Educación la evaluación, es una de las áreas más complejas, ya que sustenta que el propósito fundamental de la educación es corroborar los alcances de los objetivos previamente trazados con respecto al aprendizaje.

Lo antes mencionado puede ser una de las causas para que el estudiante no alcance el nivel satisfactorio en matemática. Cabe mencionar que Ecuador reprobó en Matemáticas en la evaluación internacional. Graves dificultades de los estudiantes ecuatorianos para desenvolverse en situaciones que requieren la capacidad de resolver problemas matemáticos arrojaron los resultados de las pruebas PISA-D 2018, en las que el Ecuador participó por primera vez. El 70,9\% de los estudiantes de Ecuador no alcanzó en Matemáticas el nivel 2, categorizado como el nivel de desempeño básico. El desempeño promedio de Ecuador fue de 377 sobre 1.000. (Publicado en el diario el Universo con fecha 26 de febrero del 2019).

Además, el propósito de este trabajo no fue criticar los modelos pedagógicos predominantes en cada Institución Educativa, sino hacer una reflexión sobre el quehacer pedagógico, para lograr transformarlo y lograr calidad educativa en los procesos de enseñanza-aprendizaje.

\section{Conclusiones.}

- En las cuatro Unidades Educativas: Pedro Vicente Maldonado, Yaruquies, Dr. Nicanor Larrea y colegio de Bachillerato Chambo, los modelos que predominan en la planificación y operatividad de la práctica docente son de tendencia conductista. El propósito del sistema educativo debe ser buscar estudiantes que cuestionen, que indaguen, que puedan gestionar positivamente el disenso, la controversia; que promuevan la inclusión, pero hasta el momento eso no ha cambiado en su totalidad.

- De la observación de clase realizada a cada docente, algunos incluían en su práctica, un modelo educativo concebido o estructurado a criterio personal de forma consciente o inconscientemente, dada la naturaleza de la Unidad Educativa.

- En base a las respuestas de la entrevista semiestructurada se puedo evidenciar que existe una carencia en cuanto a conocimiento y aplicación de los modelos pedagógicos en las instituciones educativas, debido a que se observó un poco de incomodidad al momento de tratar de explicar en qué consistían los modelos pedagógicos y sus corrientes filosóficas

\section{Referencias bibliográficas.}

- Estupiñan, N. (2012). Análisis de los modelos pedagógicos implementados en tres instituciones educativas del sector oficial de la ciudad de Santiago de Cali (Tesis de Maestría). Universidad de Colombia. Palmira-Colombia. 
- Galarza Mena \& Bethy Arlene. (2012). Realidad de la práctica pedagógica y curricular en la educación ecuatoriana en los centros educativos de básica y bachillerato de la Unidad Educativa Fe y Alegría "La Dolorosa" de la ciudad de Manta.2011-2012. (Tesis de Magister). UTPL. Manta. pp. 132.

- Hernández, R, Infante, M. (2016). El método de enseñanza-aprendizaje de trabajo independiente en la clase encuentro: recomendaciones didácticas. Revista de Pedagogía, vol. 37, Núm. 101, 2016, pp. 215-231. Universidad Central de Venezuela Caracas, Venezuela.

- Hurtado, H. (2008). Estilos de enseñanza y modelos pedagógicos: Un estudio con docentes del Programa de Ingeniería Financiera de la Universidad Piloto de Colombia. (Tesis de Magister). Universidad de La Salle Bogotá.

- Medina, J. (2013). "Modelo pedagógico y su incidencia en la evaluación del proceso enseñanza aprendizaje del Colegio Técnico Particular Nocturno "Galápagos" del Cantón Riobamba de la Provincia del Chimborazo". (Tesis de Magister). Universidad Técnica de Ambato.

- Ortíz. A. (2013). Modelos Pedagógicos. Bogotá - Colombia. Ediciones de la U. Ministerio de Educación.

- Prado, v. (2015). El modelo pedagógico como factor asociado al rendimiento de los estudiantes de educación básica primaria en las pruebas saber. Análisis hermenéutico cualitativo en la ciudad de Bogotá. (tesis doctoral). BogotáColombia.

- Cartuche, N., Tusa, M., Agüinsaca, j., Wilman Merino, Walter Tene. El modelo pedagógico en la práctica docente de las universidades públicas del país*

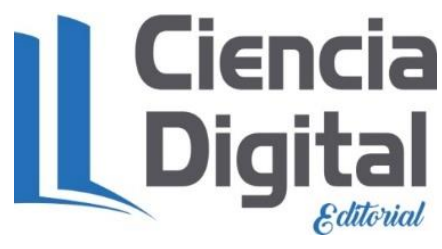


Para citar el artículo indexado.

Sánchez Salcán, N. de J., \& Londo Yachambáy, F. (2019). Identificación del modelo pedagógico matemático en la práctica docente. Explorador Digital, 3(3.1), 5-18. https://doi.org/10.33262/exploradordigital.v3i3.1.861

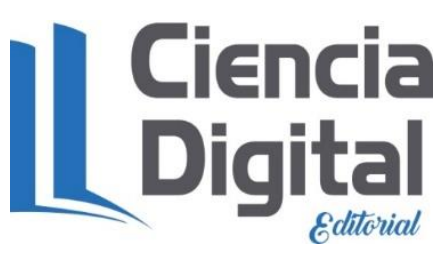

El artículo que se publica es de exclusiva responsabilidad de los autores y no necesariamente reflejan el pensamiento de la Revista Explorador Digital.

El articulo queda en propiedad de la revista y, por tanto, su publicación parcial y/o total en otro medio tiene que ser autorizado por el director o editor de la Revista Explorador Digital.
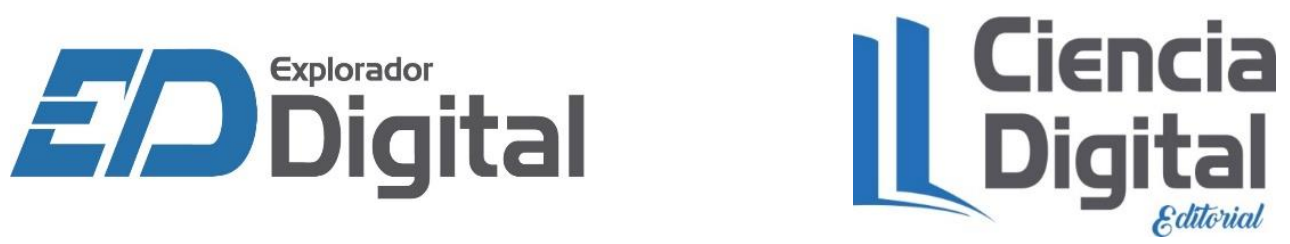